\title{
Influencia de la dieta vegetariana y carnívora en relación a la hemoglobina y hematocrito
}

Oscar Yalle-Herencia*1,a, Gonzalo Larico-Ayma 1,a , Gabrielly Boeira-De Sousa 1,a , Pool Marcos-Carbajal 2,3,b

\section{RESUMEN}

El presente identifica el grado de influencia de la dieta vegetariana y carnívora en relación con la hemoglobina y hematocrito de los estudiantes de medicina humana de la Universidad Peruana Unión, Lima, 2018. El problema planteado es descubrir si las personas con dieta vegetariana y carnívora presentan deficiencia de hematocrito y hemoglobina. En relación a las personas que llevan una dieta vegetariana, 23 participantes practican diferentes tipos de vegetarianismo. Se consideraron los siguientes rangos, hemoglobina (12-16 g/dl) y hematocrito (36-48 \%). Se concluye que, en relación a dieta y hemoglobina/hematocrito, la mayoría de los encuestados están en los rangos normales de dichas variables y que las personas que mantienen una dieta vegetariana no presentan un alto grado de deficiencia en los niveles de hematocrito y hemoglobina, y presentan un mejor nivel nutricional que los de dieta carnívora.

Palabras claves: Dieta vegetariana; Hematocrito; Anemia; Hemoglobina; Perú (Fuente: DeCS BIREME)

\section{Influence of the vegetarian and carnivore diets on hemoglobin and hematocrit levels}

\section{ABSTRACT}

The present study establishes the degree of influence of the vegetarian and carnivore diets on the hemoglobin and hematocrit levels of students of human medicine of the Universidad Peruana Unión, Lima, 2018. The goal was to find out if people who follow a vegetarian or carnivore diet are deficient in hematocrit and hemoglobin. Out of the vegetarians, 23 followed different types of vegetarianism. The following ranges were considered: hemoglobin (12 - $16 \mathrm{~g} / \mathrm{dL})$ and hematocrit (36-48\%). It is concluded that, regarding the diet and hemoglobin/hematocrit levels, most respondents show normal ranges, and people who follow a vegetarian diet do not present highly-deficient hematocrit and hemoglobin levels, and have a better nutritional level than those who follow a carnivore diet.

Keywords: Vegetarian diet; Hematocrit; Anemia; Hemoglobin; Peru (Source: MeSH NLM).

1 Universidad Peruana Unión, EP Medicina Humana. Lima, Perú.

2 Universidad Peruana Unión, EP Medicina Humana, Laboratorio de Investigación en Biología Molecular. Lima, Perú.

3 Universidad de San Martin de Porres, Facultad de Medicina, Instituto de Investigación, Centro de Investigación en Medicina de Altura.

Lima, Perú.

a Estudiante de Medicina 2do año.

b Magíster en Biología Molecular.

*Autor corresponsal 


\section{INTRODUCCIÓN}

La problemática mundial de nutrición vegetariana versus carnívora sigue siendo un tema controversial, y la principal interrogante es, ¿existe alguna desventaja al llevar una dieta vegetariana o carnívora?, y la duda más concurrente es la de "¿si soy vegetariano, tendré algún déficit con respecto a mi hemoglobina y hematocrito?" (1).

La dieta vegetariana se remonta a tiempos antiguos y fue descrita por los sabios griegos y egipcios como la dieta natural del ser humano. Con el paso del tiempo, la nutrición vegetariana ha demostrado que es mas saludable cuando está bien planificada y cubre satisfactoriamente los requerimientos energéticos y nutricionales de la persona. Los vegetarianos tienden a presentar menores valores de mortalidad y morbilidad con respecto a enfermedades crónicas degenerativas en comparación con aquellos que aún siguen una dieta a base de carnes ${ }^{(2,3)}$.

En las últimas décadas la idea de empezar una dieta vegetariana se ha expandido en nuestra sociedad. Se tiene la idea que ser o convertirse en vegetariano es sinónimo de déficit nutricional, pero si la dieta esta balanceada no habrá ningún inconveniente en el desarrollo normal de la persona.

Estudios recientes han demostrado que llevar una dieta vegetariana reduce el riesgo de enfermedades crónicas y cardiovasculares, principalmente ${ }^{(4)}$. Sin embargo, las dietas vegetarianas muy estrictas pueden aumentar el riesgo de deficiencias nutricionales.

La dieta vegetariana consta de distintos subgrupos, los mas conocidos son vagetariana, lactovegetariana, lactoovovegetariana y vegana ${ }^{(5)}$.

Dieta vegana: En esta dieta se elimina totalmente el consumo de carnes (pollo, carne, pescado, mariscos) y productos animales (leche, queso, huevos) y también la miel. Solo se permiten, granos, semillas, legumbres, hortalizas, frutas frescas y secas ${ }^{(6)}$.

Dieta lactovegetariano: Incluyen en la dieta vegetariana solo productos lácteos (leche y sus derivados) ${ }^{(4)}$.

Dieta ovovegetariano: Además de la dieta vegetariana normal vegetariana solo se permite el consumo de huevos ${ }^{(7,8)}$.

Dieta lacto-ovo-vegetariano: Semillas, legumbres, frutas verduras, etc. En esta dieta no hay consumo de carnes, pero sí de productos lácteos y huevos ${ }^{(9)}$.

Sin embargo, una de las anemias más comunes, la anemia ferropénica o megaloblástica, relacionada a un déficit nutricional de hierro y vitaminas B12 (cobalamina) y B9 (ácido fólico), presentes, principalmente, en alimentos de origen animal, representa una desventaja evidente en los vegetarianos ${ }^{(10-12)}$.

Según la evidencia científica, el principal problema en los vegetarianos es la falta de control sobre los alimentos que ingieren. En otras palabras, si agrupamos a todas las personas que no consumen carnes como vegetarianos no podríamos tener un resultado preciso. Esto sucede porque existen diferentes tipos de vegetarianismo, y en cada uno se consumen alimentos distintos que influyen en los resultados de los estudios. Por lo tanto, es imperante obtener más detalles con respecto a la dieta elegida por los encuestados, de acuerdo a los diferentes tipos de vegetarianismo ${ }^{(13)}$.

El objetivo de este trabajo es identificar el grado de influencia de la dieta vegetariana y carnívora en relación con la hemoglobina y hematocrito en los estudiantes de medicina de la Universidad Peruana Unión (Lima) en 2018. Para poder identificar la relación existente, se encuestaron a 132 estudiantes de diferentes años de la carrera de medicina, de ellos 103 son carnívoros y 29 veg etarianos. Los factores considerados fueron la dieta, genero, edad y procedencia.

Esta información analiza las diferencias con respecto a los niveles de hemoglobina $(14 \pm 2 \mathrm{~g} / \mathrm{dl}$ en mujeres y 16 $\pm 2 \mathrm{~g} / \mathrm{dl}$ en hombres) y hematocrito $(42 \pm 2 \%$ mujeres y $47 \pm 6 \%$ hombres) en relación al tipo de dieta de los estudiantes de medicina de la Universidad Peruana Unión. De esta manera, se puede tener una idea de cómo influye esta variable en la calidad de vida y prevención de enfermedades ${ }^{(14)}$.

A nivel mundial, las anemias constituyen un problema de salud. En el Perú no se ha establecido la relación que tienen con el estilo de vida de las personas.

\section{EL ESTUDIO}

El estudio es de tipo descriptivo de corte transversal. Se describe la influencia del tipo de dieta en la hemoglobina y el hematocrito de los participantes. Además, se muestra cuál de los grupos de alumnos se encuentra más afectado, según año académico. En el estudio no se manipuló ninguna variable y el instrumento de investigación se aplicó en un solo tiempo. Los investigadores resaltan la complejidad del tema por influencia del tipo de dieta sobre la hemoglobina y el hematocrito en los estudiantes de medicina, y buscan el interés de la sociedad científica para que se realicen investigaciones posteriores del mismo tema.

La población fue de 320 estudiantes de diferentes años de la carrera de medicina que participaron 
voluntariamente. De ellos, 123 fueron encuestados y, de este grupo, 103 estudiantes siguen una dieta basada en el consumo de carne, y 29 estudiantes consumen vegetales. Por el escaso número de participantes vegetarianos encuestados se optó reducir las muestras de sangre a 91 . Se justifica este hecho mediante una proporción aritmética de $3: 1$; y se asume que son 23 vegetarianos y 68 carnívoros.

\section{Estudio de muestras sanguíneas}

Cada muestra de sangre fue debidamente extraída y analizada para hallar el valor del hematocrito mediante centrifugación. Este método es considerado el gold estándar en muchos laboratorios y consiste en centrifugar a alta velocidad la muestra de sangre para separarla en 3 capas. La primera (superficial) está compuesta por plasma; la siguiente, por células blancas y plaquetas; y, por último, la capa más inferior está conformada por glóbulos rojos, y el espesor es el que determina el nivel de hematocrito ${ }^{(15,16)}$.

Las muestras se tomaron en el Laboratorio de Microbiología. El pinchazo se realizó con una lanceta y la sangre se colocó en un capilar. Las muestras se centrifugaron y se obtuvo el hematocrito $y$, en consecuencia, la hemoglobina.

Los elementos referentes al tipo de dieta (basada en carne o vegetales) se evaluaron mediante una encuesta conformada por preguntas de opción múltiple, distribuidas en datos generales y hábitos alimentarios. Los datos de la encuesta y las variables se analizaron con programa estadístico IBM SPSS versión 22 .

Esta investigación fue aprobada por el Comité de Ética institucional de la Universidad Peruana Unión.

\section{RESULTADOS}

\section{Análisis descriptivo sociodemográficos}

Presentamos datos sociodemográficos con el propósito de contrastarlos y para obtener datos descriptivos relevantes, y observar el comportamiento de los mismos y llegar a conclusiones importantes para tomar decisiones en la entidad estudiada.

En relación con el hematocrito, se observa que los participantes que tienen una dieta carnívora presentan un hematocrito disperso en los rangos de 40-45. Por otro lado, en los vegetarianos se presentaron valores más centrados en los rangos considerados normales (10 de los 22 encuestados) y, por último, el único participante que es vegano mostró un nivel de hematocrito por encima de lo establecido, lo que muestra un resultado óptimo para nuestro estudio (Tabla 1 ).

Tabla 1. Relación del tipo de dieta y el hematocrito de los estudiantes en estudiantes de medicina (Universidad Peruana Unión)

\begin{tabular}{|c|c|c|c|c|c|c|c|c|c|c|}
\hline \multicolumn{11}{|c|}{$\begin{array}{l}\text { Dieta y hematocrito en estudiantes de medicina } \\
\text { Hematocrito \% }\end{array}$} \\
\hline & & & $34-36$ & $37-39$ & $40-42$ & $43-45$ & $46-48$ & 49-51 & $55-57$ & Total \\
\hline \multirow[t]{6}{*}{ Dieta } & \multirow[t]{2}{*}{ Consumo de carne } & Recuento & 2 & 6 & 17 & 17 & 12 & 6 & 4 & 68 \\
\hline & & $\%$ del total & 2,20 & 6,60 & 18,70 & 18,70 & 13,20 & 6,60 & 4,40 & 74,70 \\
\hline & \multirow{2}{*}{ Vegetarianos } & Recuento & 1 & 1 & 4 & 10 & 6 & 0 & 0 & 22 \\
\hline & & $\%$ del total & 1,10 & 1,10 & 4,40 & 11,00 & 6,60 & 0,00 & 0,00 & 24,20 \\
\hline & & Recuento & 0 & 0 & 0 & 0 & 1 & 0 & 0 & 1 \\
\hline & Vegano & $\%$ del total & 0,00 & 0,00 & 0,00 & 0,00 & 1,10 & 0,00 & 0,00 & 1,10 \\
\hline \multirow[t]{2}{*}{ Total } & & Recuento & 3 & 7 & 21 & 27 & 19 & 6 & 4 & 91 \\
\hline & & $\%$ del total & 3,30 & 7,70 & 23,10 & 29,70 & 20,90 & 6,60 & 4,40 & 100,00 \\
\hline
\end{tabular}

Al relacionar los valores de hemoglobina con los tipos de dieta se observa que los vegetarianos presentan un mejor porcentaje (valores considerados normales). Por otro lado, las personas que consumen carnes presentan una mayor variedad de resultados y un porcentaje considerable en el rango de 11-13 (Tabla 2). 
Influencia de la dieta vegetariana y carnívora en relación a la hemoglobina y hematocrito

Tabla 2. Relación del tipo de dieta y la hemoglobina en estudiantes de medicina (Universidad Peruana Unión)

\begin{tabular}{|c|c|c|c|c|c|c|}
\hline \multicolumn{7}{|c|}{$\begin{array}{l}\text { Dieta y hemoglobina en estudiantes de Medicina } \\
\qquad \text { Hemoglobina } \mathrm{g} / \mathrm{dl}\end{array}$} \\
\hline & & & $11-13$ & $14-16$ & $17-19$ & Total \\
\hline \multirow[t]{6}{*}{ Dieta } & \multirow{2}{*}{$\begin{array}{l}\text { Personas que } \\
\text { consumen carnes }\end{array}$} & Recuento & 21 & 38 & 9 & 68 \\
\hline & & $\%$ del total & 23,10 & 41,80 & 9,90 & 74,70 \\
\hline & \multirow[t]{2}{*}{ Vegetarianos } & Recuento & 5 & 17 & 0 & 22 \\
\hline & & $\%$ del total & 5,50 & 18,70 & 0,00 & 24,20 \\
\hline & \multirow[t]{2}{*}{ Vegano } & Recuento & 0 & 1 & 0 & 1 \\
\hline & & $\%$ del total & 0,00 & 1,10 & 0,00 & 1,10 \\
\hline \multirow[t]{2}{*}{ Total } & & Recuento & 26 & 56 & 9 & 91 \\
\hline & & $\%$ del total & 28,60 & 61,50 & 9,90 & 100,00 \\
\hline
\end{tabular}

El valor promedio del hematocrito es $44 \%$. La mayoría de los participantes muestran valores en el rango normal de 43-45, lo que muestra un mejor resultado estadístico (Tabla 3).

Tabla 3. Nivel de hematocrito, según año académico

\begin{tabular}{|c|c|c|c|c|c|c|c|c|c|}
\hline \multicolumn{10}{|c|}{$\begin{array}{c}\text { Hematocrito y año académico } \\
\text { Hematocrito \% }\end{array}$} \\
\hline Año & & $34-36$ & $37-39$ & $40-42$ & $43-45$ & $46-48$ & $49-51$ & $52-54$ & $55-57$ \\
\hline \multirow[t]{2}{*}{$2^{\circ}$ (grupo 1) } & Recuento & 2 & 2 & 8 & 13 & 2 & 1 & 1 & 3 \\
\hline & $\%$ del total & 2,20 & 2,20 & 8,80 & 14,30 & 2,20 & 1,10 & 1,10 & 3,30 \\
\hline \multirow[t]{2}{*}{$2^{\circ}$ (grupo 2) } & Recuento & 0 & 0 & 3 & 4 & 2 & 2 & 0 & 0 \\
\hline & $\%$ del total & 0,00 & 0,00 & 3,30 & 4,40 & 2,20 & 2,20 & 0,00 & 0,00 \\
\hline \multirow[t]{2}{*}{$3^{\circ}$} & Recuento & 0 & 1 & 3 & 2 & 4 & 3 & 0 & 0 \\
\hline & $\%$ del total & 0,00 & 1,10 & 3,30 & 2,20 & 4,40 & 3,30 & 0,00 & 0,00 \\
\hline \multirow[t]{2}{*}{$4^{\circ}$} & Recuento & 1 & 2 & 3 & 5 & 5 & 0 & 3 & 1 \\
\hline & $\%$ del total & 1,10 & 2,20 & 3,30 & 5,50 & 5,50 & 0,00 & 3,30 & 1,10 \\
\hline \multirow[t]{2}{*}{$5^{\circ}$} & Recuento & 0 & 0 & 1 & 0 & 2 & 0 & 0 & 0 \\
\hline & $\%$ del total & 0,00 & 0,00 & 1,10 & 0,00 & 2,20 & 0,00 & 0,00 & 0,00 \\
\hline \multirow[t]{2}{*}{$6^{\circ}$} & Recuento & 0 & 2 & 3 & 3 & 4 & 0 & 0 & 0 \\
\hline & $\%$ del total & 0,00 & 2,20 & 3,30 & 3,30 & 4,40 & 0,00 & 0,00 & 0,00 \\
\hline \multirow[t]{2}{*}{ Total } & Recuento & 3 & 7 & 21 & 27 & 19 & 6 & 4 & 4 \\
\hline & $\%$ del total & 3,30 & 7,70 & 23,10 & 29,70 & 20,90 & 6,60 & 4,40 & 4,40 \\
\hline
\end{tabular}

En relación al hematocrito, el 2. ${ }^{\circ}$ año-grupo 1 presenta el mayor porcentaje en rango de 43-45. Por otro lado, se observa que el 2,2 \% del mismo año y grupo no tienen un adecuado valor de hematocrito. 
Tabla 4. Nivel de hemoglobina, según año académico

\begin{tabular}{|c|c|c|c|c|c|}
\hline \multicolumn{6}{|c|}{$\begin{array}{c}\text { Hemoglobina y año académico } \\
\text { Hemoglobina } \mathrm{g} / \mathrm{dl}\end{array}$} \\
\hline Año & & $11-13$ & $14-16$ & $17-19$ & Total \\
\hline \multirow[t]{2}{*}{$2^{\circ}$ (grupo 1) } & Recuento & 11 & 17 & 4 & 32 \\
\hline & $\%$ del total & 12,10 & 18,70 & 4,40 & 35,20 \\
\hline \multirow[t]{2}{*}{$2^{\circ}$ (grupo 2) } & Recuento & 3 & 8 & 0 & 11 \\
\hline & $\%$ del total & 3,30 & 8,80 & 0,00 & 12,10 \\
\hline \multirow[t]{2}{*}{$3^{\circ}$} & Recuento & 2 & 10 & 1 & 13 \\
\hline & $\%$ del total & 2,20 & 11,00 & 1,10 & 14,30 \\
\hline \multirow[t]{2}{*}{$4^{\circ}$} & Recuento & 6 & 10 & 4 & 20 \\
\hline & $\%$ del total & 6,60 & 11,00 & 4,40 & 22,00 \\
\hline \multirow[t]{2}{*}{$5^{\circ}$} & Recuento & 0 & 3 & 0 & 3 \\
\hline & $\%$ del total & 0,00 & 3,30 & 0,00 & 3,30 \\
\hline \multirow[t]{2}{*}{$6^{\circ}$} & Recuento & 4 & 8 & 0 & 12 \\
\hline & $\%$ del total & 4,40 & 8,80 & 0,00 & 13,20 \\
\hline \multirow[t]{2}{*}{ Total } & Recuento & 26 & 56 & 9 & 91 \\
\hline & $\%$ del total & 28,60 & 61,50 & 9,90 & 100,00 \\
\hline
\end{tabular}

\section{DISCUSIÓN}

Los participantes siguen diferentes tipos de dietas. Las más frecuentes son vegetarianismo y el consumo de carne. Se ha observado que los niveles bajos de hematocrito y hemoglobina se encuentran en el grupo que consume carne. Esto refuta, en parte, la idea de que los vegetarianos presentan anemia con más frecuencia (por la deficiencia de vitamina B12). Lo más importante es complementar los alimentos para un mejor control de la salud y para evitar deficiencias nutricionales.

Se observó que la mayoría de estudiantes presentan un hematocrito en niveles normales, debido al hecho que la mayoría mantiene una dieta rica en fuentes tanto animales como vegetales.

Los parámetros del índice de Quetelet o índice de masa corporal (IMC) se encontraron en valores normales (18-24,9). En el estudio también se tomaron en cuenta algunas enfermedades que podían alterar los resultados como cuadros cardiorrespiratorios como enfermedad obstructiva crónica pulmonar (EPOC), asma o ateroesclerosis, lo que finalmente, no mostró ninguna alteración (17).

Los estudiantes que cursan el segundo año de la carrera de medicina están mejor informados sobre nutrición y, por esa razón, presentaron los niveles más altos de hematocrito y hemoglobina. Sin embargo, los alumnos de otros años (quinto y sexto) no se encontraban en las mejores condiciones; esto se debe al estrés que experimentan por las prácticas en los hospitales, lo que influye también en los niveles de hematocrito y hemoglobina ${ }^{(18)}$.

De los 132 encuestados, en la relación entre año académico y el vegetarianismo predominaron los alumnos del segundo año-grupo 1 , debido a que este es el grupo más numerosos $y$, por consiguiente, presentan un mayor número de resultados válidos.

Los consumidores de carne presentan un número importante de antecedentes familiares de cuadros como diabetes y EPOC, en comparación con los vegetarianos. Los casos de hipertensión y diabetes son los más frecuentes en los estudiantes con dieta carnívora. Un estudio que relacionó el tipo de dieta y las enfermedades cardiovasculares reportó que ingerir carnes rojas procesadas aumenta el riesgo de desarrollar hipertensión arterial (HTA) (20-40\%), en comparación con el consumo de carnes rojas sin procesar ${ }^{(19)}$.

Los alumnos vegetarianos muestran también 
antecedentes de enfermedades, estos problemas de salud derivan de la dieta vegetariana o son la razón del cambio a este régimen. Se observó que un pequeño grupo presentó el antecedente de anemia megaloblástica, cuadro en que las concentraciones de homocisteína son elevadas y que está presente en los vegetarianos. Destaca el caso de un estudiante que presenta HTA y, en consecuencia, un factor de riesgo de arteriosclerosis coronaria, cerebral, periférica y aórtica ${ }^{(20,21)}$.

Se concluye que las personas que mantienen una dieta vegetariana no presentan bajos niveles de hematocrito y hemoglobina, lo que contradice la idea que se tiene del tema.

Como limitaciones del estudio podemos mencionar la ausencia del volumen corpuscular medio (VCM) para complementar la investigación, la escasa información de otras fuentes bibliográficas y la falta de cooperación de algunos estudiantes.

Este estudio presenta la primera evidencia de relación entre las dietas vegetariana y carnívora con niveles de hemoglobina y hematocrito en nuestro país, ya que no se ha mostrado en otras fuentes esta relación.

Agradecimiento: A los estudiantes y docentes de la EP de Medicina Humana de la Universidad Peruana Unión por brindarnos los materiales, infraestructura y participación en este estudio.

Contribución de los autores: P. Marcos ha participado en la concepción y diseño del artículo. Los procedimientos y resultados fueron realizados por 0 . Yalle y $G$. Larico. Los análisis y discusiones fueron realizados por $P$. Marcos, O. Yalle, G. Larico y G. Boeira. La redacción del artículo estuvo a P. Marcos, O. Yalle, G. Larico y G. Boeira. La revisión crítica la realizó P. Marcos. La versión final estuvo a cargo de P. Marcos, O. Yalle, G. Larico y G. Boeira.

Fuentes de financiamiento: Este artículo ha sido financiado por el Laboratorio de Investigación en Biología molecular de la EP Medicina Humana de la Facultad de Ciencias de la Salud de la Universidad Peruana Unión (UPeU).

Conflicto de interés: Los autores declaran no tener ningún conflicto de interés.

\section{REFERENCIAS BIBLIOGRÁFICAS}

1. Organización Mundial de Salud. Concentraciones de hemoglobina para diagnosticar la anemia y evaluar su gravedad [Internet]. 2019. Disponible en: https://www.who.int/vmnis/indicators/ haemoglobin/es/
2. Suazo Pulgar GP. Vegetarianismo, la opción para una mejor calidad de vida, desde la perspectiva de la comunidad Yoguica. Un Estudio Cualitativo [Tesis]. Valdivia: Universidad Austral de Chile. Facultad de Medicina; 2007.

3. Saz Peiro P. Historia del vegetarianismo [Internet]. 2019. Disponible en: http://www.unizar.es/med_naturista/historia\%20 y\%20dieta\%20vegetariana.pdf

4. López Portillo L, García Campos M, Montijo Barrios E, Cervantes Bustamante R, Mata Rivera N, Ramírez Mayans J. La dieta vegetariana en los niños. Ventajas, desventajas y recomendaciones dietéticas. Acta Pediatr Mex. 2006; 27(4): 205-12.

5. Martínez Biarge M. Niños vegetarianos, ¿niños sanos?. Actualización en Pediatría. 2017; 2017: 253-68.

6. Astudillo Rosero EP. Relación entre los niveles de hemoglobina y hematocrito y los hábitos alimentarios de diferentes tipos de personas vegetarianas que asisten a Ming Yuen restaurante vegetariano en mayo 2013 [Tesis]. Quito: Pontificia Universidad Católica del Ecuador. Facultad de Enfemería; 2013.

7. Gonzalez Miranda DM. Estudio exploratorio del vegetarianismo en adultos de 20 a 50 años de edad en la ciudad de Guatemala [Tesis]. Guatemala de la Asunción: Universidad Rafael Landívar. Facultad de Ciencias de la Salud; 2014.

8. Serralde Zúñiga AE, Pasquetti Ceccatelli A, Meléndez Mier G. Micronutrimentos en vegetarianos. Rev Endocrinol y Nutr. 2005; 13(1): 33-8.

9. Domínguez Álvarez R. Un análisis de las personas vegetarianas en la Universidad de La Laguna. Federación Español de Sociología. 2016.

10. Varela-Moreiras G, Alonso Aperte E. Ácido fólico y salud. Madrid: Fundación Española de Nutrición; 1999. Disponible en:https://www. fen.org.es/storage/app/media/imgPublicaciones/15220074224.pdf

11. Comité Nacional de Hematologí; Oncología y Medicina Transfucional; Comité Nacionl de Nutrición. Deficiencia de hierro y anemia ferropénica. Guía para su prevención, diagnóstico y tratamiento. Arch Argent Pediatr. 2017; 115(4): s68-82.

12. Ministerio de Salud del Perú. Guía de Práctica Clínica para el Diagnóstico y Tratamiento de la Anemia por deficiencia de Hierro en niñas, niños y adolescentes en establecimientos de Salud del primer nivel de Atención [Internet]. Disponible en: http://bvs. minsa.gob.pe/local/MINSA/3932.pdf.

13. Sociedad Argentina de Nutrición. Alimentación vegetariana: posición de la Sociedad Argentina de Nutrición [Internet]. 2014. Disponible en: http://www.sanutricion.org.ar/files/upload/files/ Alimentacion_Vegetariana_Posicion_SAN.pdf

14. Unión Vegetariana Española. Introducción a las dietas vegetarianas [Internet]. Union Vegetariana Española. 2017. Disponible en: https://unionvegetariana.org/introduccion-a-lasdietas-vegetarianas/

15. Cegarra Sanmartín V. Comparación de tres métodos de medición de hemoglobina en cirugía cardíaca [Internet]. Universidad Autónoma de Barcelona; 2012. Disponible en: https://ddd.uab.cat/pub/ trerecpro/2012/hdl_2072_203376/TR-CegarraSanmartin.pdf

16. Campuzano Maya G. Del hemograma manual al hemograma de cuarta generación. Medicina y Laboratorio. 2007; 13(11-12): 511-50.

17. Pajuelo J, Amemiya I. El uso del índice de Quetelet en el diagnóstico nutricional en niños. An Fac Med. 1996; 57(2): 103-8.

18. Sánchez Jiménez A. Ferropenia: impacto sobre el estado de salud mental [Tesis]. Asturias: Universidad de Oviedo. Facultad de Medicina; 2015.

19. Gutiérrez Fuentes JA, Gómez Gerique JA, Gómez de la Camara A. Dieta y riesgo de enfermedades cardiovasculares en España. Med Clin Monogr. 2011; 12: 1-39.

20. Castells Bescós E, Boscá Crespo AR, García Arias C, Sánchez 
Chaparro MA. Hipertensión Arterial [Internet]. Disponible en: http: / / www. medynet.com/usuarios/jraguilar/Manual\%20de $\% 20$ urgencias $\% 20 y \% 20$ Emergencias/htaul.

21. Coon RW. Elena G. de White y el vegetarianismo. Revista Adventista. 1986; 1-25.

\section{Correspondencia:}

Oscar Joel Yalle Herencia

Dirección: Alameda de Ñaña, manzana L, lote 12. Chosica.

Lima, Perú.

Teléfono: 931577737

Correo electrónico: oscaryalle@upeu.edu.pe

Recibido: 13 de marzo de 2019

Evaluado: 25 de marzo de 2019

Aprobado: 17 de julio de 2019 (c) La revista. Publicado por Universidad de San Martín de Porres, Perú. (c) Br Licencia de Creative Commons Artículo en acceso abierto bajo términos de Licencia Creative Commons Atribución 4.0 Internacional. (http://creativecommons.org/licenses/by/4.0/)

ORCID iDs

Oscar Yalle $\quad$ https://orcid.org/0000-0001-8443-0208

Gonzalo Larico https://orcid.org/0000-0001-5208-8022

Gabrielly Boeira 1 https://orcid.org/0000-0002-5789-0687

Pool Marcos 1 https: / / orcid.org/0000-0002-7741-0337 